\title{
Quantum Tunneling Characteristics in Monolayer Graphene Modulated by Multiple Electrostatic Barriers
}

\author{
Hassen Dakhlaoui ${ }^{1,2, *}$, Walid Belhadj ${ }^{3}$, Bryan M. Wong ${ }^{4, *}$ \\ ${ }^{1}$ Nanomaterials Technology unit, Basic and Applied Scientific Research Center (BASRC), College of Science of \\ Dammam, Imam Abdulrahman Bin Faisal University, P. O. Box 1982, 31441 Dammam, Saudi Arabia. \\ ${ }^{2}$ Department of Physics, College of Sciences for Girls, Imam Abdulrahman Bin Faisal University, Saudi Arabia \\ ${ }^{3}$ Department of Physics, College of Applied Sciences, Umm Al-Qura University, Mecca, Saudi Arabia \\ ${ }^{4}$ Department of Chemical \& Environmental Engineering, Materials Science \& Engineering Program, and Department \\ of Physics \& Astronomy, University of California-Riverside, Riverside, CA, USA
}

Corresponding Author e-mail: bryan.wong@ucr.edu

\begin{abstract}
The transmission coefficient and electronic conductance of a graphene monolayer in the presence of multi-electrostatic barriers are theoretically investigated using the transfer matrix method (TMM). The transmission coefficient, conductance, and Fano factor are evaluated as a function of the number and width of the barriers, angle/energy of incidence, as well as the applied potential at each barrier. We find that the transmission coefficient presents a series of resonances that depends on the number and widths of the barriers. Furthermore, we show that the resonant states can be suppressed for larger incidence angles and barrier widths and tuned towards lower energies. Consequently, the proposed structure can be used to fabricate new optoelectronic devices based on (ON/OFF) states as tunable field-effect transistors.
\end{abstract}

\section{1-Introduction}

The electronic and optical properties of heterostructures based on single and multilayered graphene continue to garner immense interest [1-5,31-33] due to their usage in various optoelectronic devices such as photonic sensors, graphene-based memory, solar cells, and highspeed electron transistors. In a graphene monolayer, the motion of electrons is described by the massless Dirac equation, which exhibits a linear energy dispersion. This linearity in the dispersion energy relation enables a low conductivity due to a vanishing bandgap near the Dirac point [6]. Unlike conventional materials, heterostructures based on monolayer graphene exhibit a Klein tunneling phenomenon which predicts that an electron can be transmitted across thick and high barriers with a transmission probability equal to $100 \%$ for normal incidence. Recently, the electronic transmission and conductance of Dirac electrons across single and double barriers have been investigated [7-9]. In addition, many studies have shown that an applied delta magnetic field can confine electrons in a graphene monolayer [10-14]. Additional theoretical and experimental studies have probed electron transmission through mono and bilayer graphene modulated by multiple electrostatic barriers, which have resulted in new devices based on wavevector filters and tunneling magnetoresistance effects [15-23]. Encouraged by these previous studies, this work investigates the transmission coefficient and electronic conductance produced by monolayer 
graphene under the influence of multiple electrostatic barriers. These barriers are realized by depositing various gates on top of the graphene layer. The effects of the incidence angle, number, and widths of the barriers on the transmission coefficient, conductance, and Fano factor are investigated using the transfer matrix method (TMM). The paper is organized as follows: In Section 2 , we outline the model and theoretical equations that govern electron transport with the numerical methods used for their solution. Section 3 presents, discusses, and analyzes the results of our calculations. Finally, we give our conclusions in section 4.

\section{2- Theory}

The system under consideration is a graphene monolayer in the $x y$-plane. On top of this monolayer, we have applied $N$ electrostatic potentials $(N=1, \ldots, 10)$, as shown in figures 1 (a-b). The widths of the barriers are $b_{i}(i=1, . ., N)$, and the distances separating the barriers are denoted by $a_{i}(i=1, . ., N)$. The individual potentials, $V_{i}(i=1, . ., N)$, denote the barrier heights. The motion of electrons is described by the massless Dirac equation as follows [5, 24]:

$$
\left[-i \hbar v_{f} \sigma_{x, y} \nabla+V_{j}(x)\right] \Psi(x, y)=E \Psi(x, y)
$$

In equation (1), $v_{f}$ represents the Fermi velocity, $\sigma_{x, y}$ denotes the Pauli matrices, and $E$ and $\Psi(x, y)$ are the energy and its wavefunction, respectively. The wavefunction in layer $j$ describing the motion of an incident electron with an angle of incidence $\theta$ is given by the following expression [25]:

$$
\Psi_{j}(x, y)=\left\{\begin{array}{l}
A_{j} e^{i q_{j} x}+B_{j} e^{-i q_{j} x} \\
\frac{q_{j}+i k_{y}}{k_{j}} A_{j} e^{i q_{j} x}-\frac{q_{j}-i k_{y}}{k_{j}} B_{j} e^{-i q_{j} x}
\end{array}\right\} e^{i k_{y} y}
$$

where $A_{j}$ and $B_{j}$ are the amplitudes of the wavefunction in layer $j, k_{y}=\left(E / \hbar v_{f}\right) \sin (\theta)$ denotes the $y$-component of the incident wavevector, $k_{j}=\left(E-V_{j}\right) / \hbar v_{f}$ represents the total wavevector in layer $j$, and $q_{j}=\sqrt{k_{j}^{2}-k_{y}^{2}}$. Using the continuity of the wavefunction at each interface separating two consecutive layers, we can readily obtain the matrix relating the amplitudes of the wavefunctions in the first and last layers as follows [26-28]:

$$
\left(\begin{array}{l}
A_{1} \\
B_{1}
\end{array}\right)=S\left(\begin{array}{l}
A_{N} \\
B_{N}
\end{array}\right)
$$

Where $S$ represents the transfer matrix given by:

$$
S=\left(\begin{array}{ll}
S_{11} & S_{12} \\
S_{21} & S_{22}
\end{array}\right)=\left(\begin{array}{cc}
1 & 1 \\
\alpha_{1} & -\beta_{1}
\end{array}\right)^{-1} \prod_{j=2}^{N-1} S_{j}\left(\begin{array}{cc}
1 & 1 \\
\alpha_{N} & -\beta_{N}
\end{array}\right)
$$

In the last equation, the coefficients $\alpha_{j}$ and $\beta_{j}$ are given by:

$$
\begin{aligned}
& \alpha_{j}=\left(q_{j}+i k_{y}\right) / k_{j} \\
& \beta_{j}=\left(q_{j}-i k_{y}\right) / k_{j}
\end{aligned}
$$


The transfer matrix between two consecutive layers $j$ and $j+1$ is given by:

$$
S_{j}=\left(\begin{array}{cc}
1 & e^{i a q_{j}} \\
\alpha_{j} & -\beta_{j} e^{i a q_{j}}
\end{array}\right)\left(\begin{array}{cc}
e^{i a q_{j}} & 1 \\
\alpha_{j} e^{i a q_{j}} & -\beta_{j}
\end{array}\right)^{-1}
$$

Using these expressions, the transmission coefficient can be calculated from the expresssion $T(E, \theta)=\left|A_{N}\right|^{2}$. The conductance at zero temperature can then be calculated using the LandauerButtiker expression [29]:

$$
G=G_{0} \int_{-\pi / 2}^{\pi / 2} T(E, \sin \theta) \cos (\theta) d \theta
$$

where $G_{0}=2 e^{2} E_{F} L_{y} /(\pi h)$, and $L_{y}$ denotes the graphene layer along the y-axis.

The Fano factor is given by the following expression [30]:

$$
F=\frac{\int_{-\pi / 2}^{\pi / 2} T[1-T] \cos (\theta) d \theta}{\int_{-\pi / 2}^{\pi / 2} T \cos (\theta) d \theta}
$$

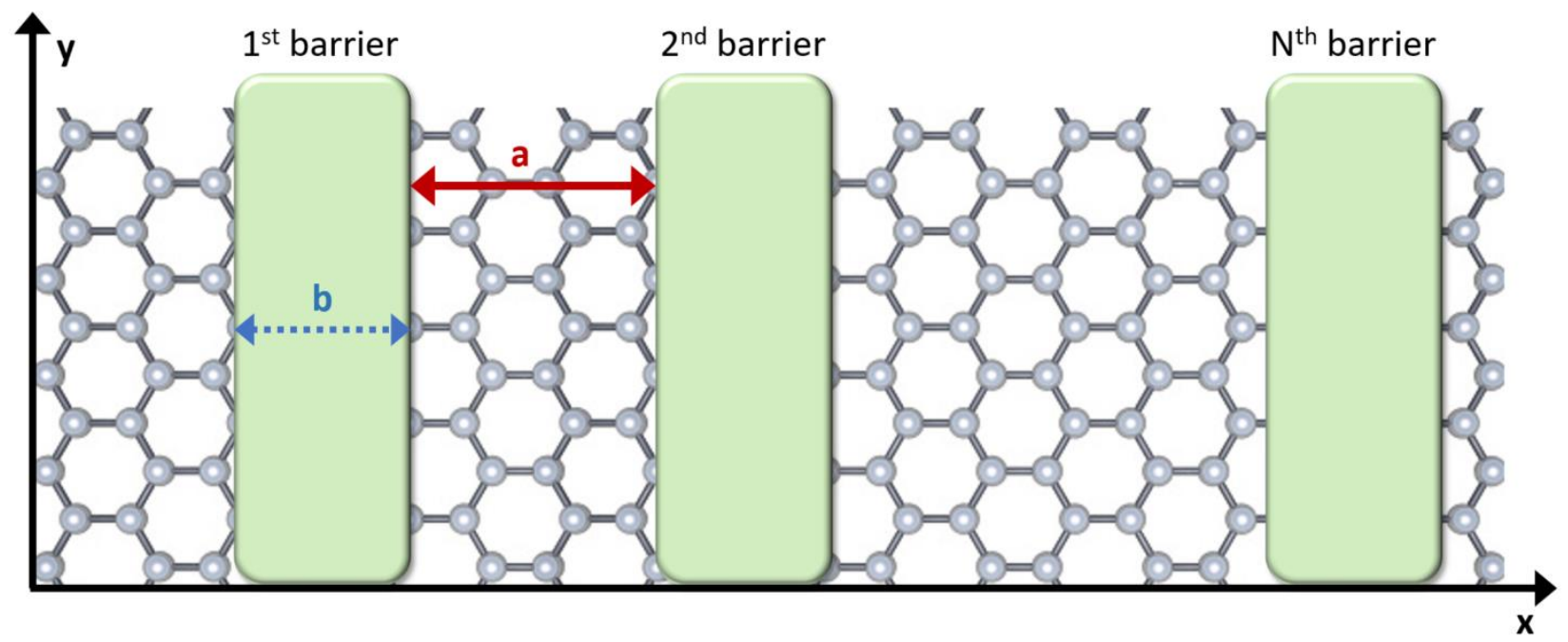

Figure 1: Schematic diagram of a graphene monolayer under external electrostatic potentials. The barrier and channel widths are denoted by $b$ and $a$, respectively.

\section{Results and discussion}

We first discuss the effect of the number of electrostatic barriers on the transmission coefficient. Figs. 2 (a-f) depicts the transmission coefficient as a function of the incident energy for three angles of incidence $(\theta=0, \theta=\pi / 6$, and $\theta=\pi / 3)$. The number of barriers varies from $N=1-10$. In this case, the widths of the barriers and wells are $b=2 \mathrm{~nm}$ and $a=2 \mathrm{~nm}$, respectively. The Fermi velocity is $v_{f}=10^{6} \mathrm{~m} / \mathrm{s}$, and the barrier heights are fixed at $V_{i=1, ., N}=0.1 \mathrm{eV}$. By examining these 
figures, we observe that the transmission coefficient presents a series of oscillations indicating the existence of resonant states (a miniband of energies) in the quantum well regions sandwiched between the barriers. Once the energy of an incident electron coincides with a resonant state, the transmission coefficient reaches its maximum. The quantum tunneling phenomenon can be explained as follows: When the energy of the incident electron is equal to the resonant eigenenergy of the quantum wells sandwiched between the barriers, the electron wave is trapped in the quantum well regions, reflecting back and forth between the barriers in such a phase as to produce constructive interference.

For the case of $\theta=0$, the transmission coefficient is equal to unity regardless of the incident energy and the number of electrostatic barriers. This behavior confirms the Klein-tunneling effect, which states that the system is fully transparent for normal incidence even for high and large barrier widths. In addition, when $\theta$ increases, the number of resonant states is reduced, and the peaks of the transmission coefficient become sharper.

Also, by examining figs 2 (b-f), we note that when the number of electrostatic barriers is increased, more resonant states are obtained. For instance, considering the case of $\theta=\pi / 6$, we observe that when the number of barriers is varied from $N=2$ to 10 , three series of resonances (red curves) emerge for the transmission coefficient. The first series of resonances contains $N$ resonant states; however, the second and the third series contain $\mathrm{N}-1$ resonant states. Another important point that should be noted is that for all these figures, the energy gaps separating the first, second, and third series of resonance are not affected by increasing the number of barriers. However, when the angle is increased $(\theta=\pi / 3)$, the second and third series of resonances are suppressed, and only one series of resonances is obtained containing resonance states whose number is equal to the number of barriers. We can conclude that the number of series of resonances (also called minibands of transmission) can be reduced by selecting high angles of incidence. As such, $\theta=\pi / 3$ is a suitable incident angle for which incident energies with high energy are completely reflected, and only electrons of the first series of resonances are transmitted. All these results confirm that the transmission coefficient can be modulated by adjusting the angle of incidence and energy of incident electrons.

To provide more mechanistic information about the transmission coefficient, figure 3 plots its variation as a function of the incident energy for three barrier widths ( $b=10,15$, and $20 \mathrm{~nm}$ ). In this case, the number of barriers is fixed at $N=5$. For $\theta=\pi / 3$, the transmission coefficient presents two series of resonances. The number of resonant states in the first series of resonance increases with the barrier width and shifts towards higher energies. However, the number of energy levels of the second series of resonances are reduced the barrier widths are increased. For instance, concerning the second series of resonances, we observe four states for $b=10 \mathrm{~nm}$, and these states are suppressed and become closer to each other for $b=15 \mathrm{~nm}$ and finally disappear completely for $b=20 \mathrm{~nm}$. This phenomenon is notable since the transmission coefficient can also be modulated by enlarging the width of the barriers and selecting the appropriate angle of incidence.

In the same way, we have also evaluated the impact of the barrier width for $\theta=\pi / 6$. For this low incidence, the transmission coefficient presents three series of resonances; however, contrary to the previous case, these series of resonances remain when the barrier width is increased. In 
addition, we observe that the energy gaps separating two consecutive series of resonant levels increase with the barrier width. All of these results provide different alternatives to modulate the operation of a spin-field transistor.
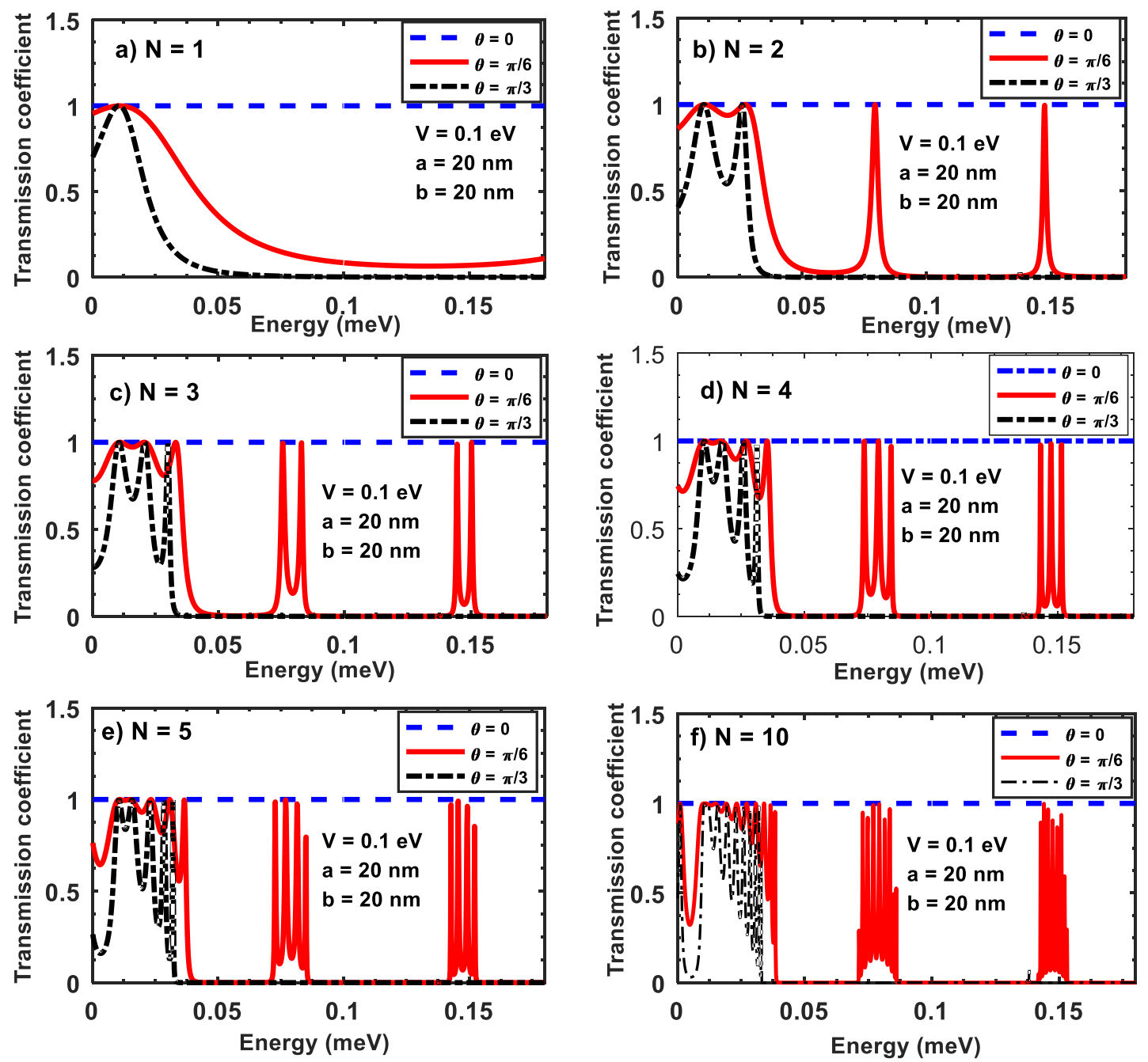

Figure 2. Variation of the transmission coefficients as a function of the incident energy for different barrier numbers. a) $N=1$; b) $N=2$; c) $N=3$, d) $N=4$, e) $N=5$, and f) $N=10$. 

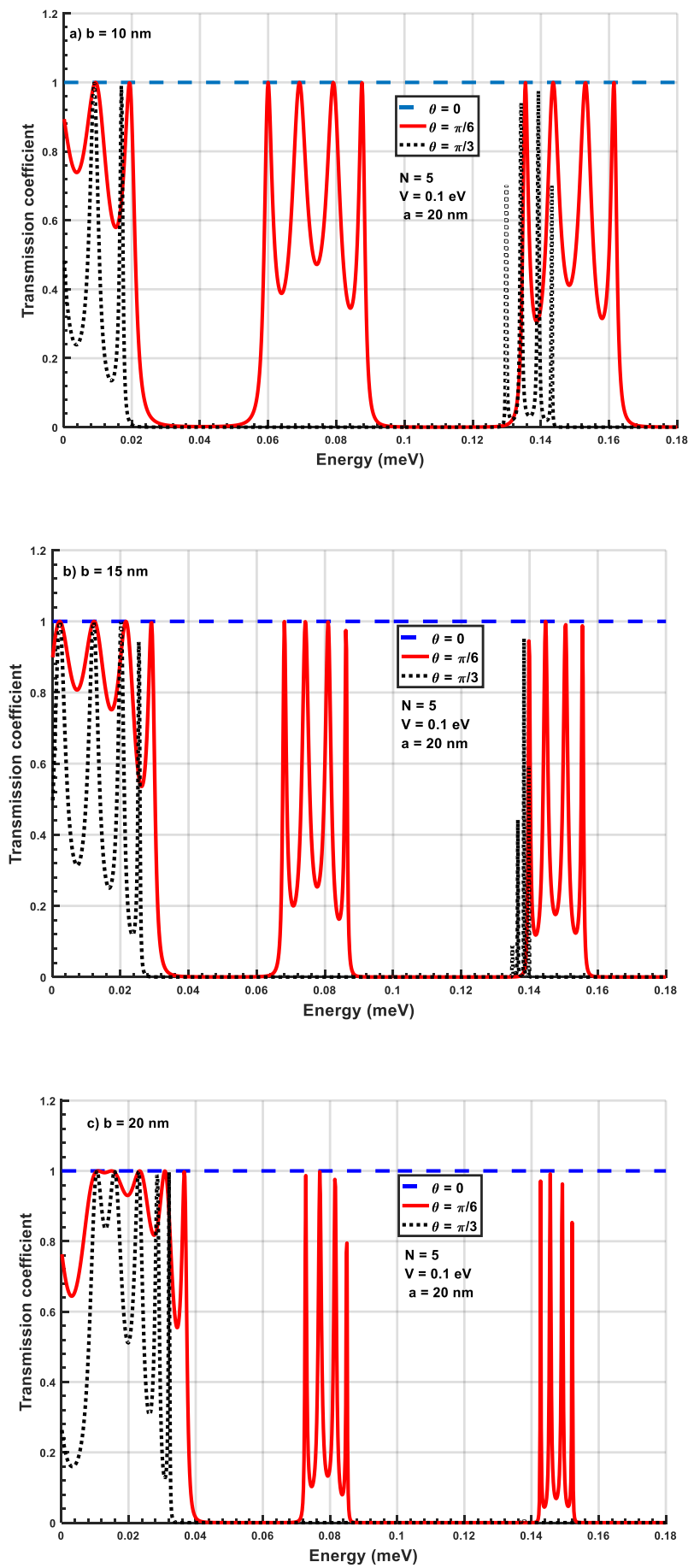

Figure 3. Variation of the transmission coefficients as a function of the incident energy for different barrier widths. a) $b=10 \mathrm{~nm}$, b) $b=15 \mathrm{~nm}$, and c) $b=20 \mathrm{~nm}$.

To further evaluate the impact of the incidence angle $\theta$ on the tunneling of the electrons through our structure, we examine the transmission coefficient as a function of $\theta$ for different numbers and 
widths of barriers. In figures 4 (a-e), we consider four cases $(N=1,2,5$, and 10). From all these figures, we observe that the transmission curves are symmetric with respect to the normal axis $(\theta=0)$ regardless of the number and widths of barriers. When the number of the barriers increases $(N>1)$, the transmission coefficient presents three series of resonances separated by two gaps. The central series of resonances occur for angles between $-0.5 \leq \theta \leq 0.5$. In addition, in this region of incidence, the transmission coefficients plateau close to $\theta=0$ and show secondary resonance peaks near unity. The number of these peaks increases with the number of barriers. The central series of resonances is surrounded by two gaps that are due to evanescent waves across the barriers. We note that these gaps increase with the thickness of the barriers, while their depths are independent of the number and widths of barriers. Furthermore, we observe that the two series of resonances located around $\theta=-1$ and $\theta=1$ are perfectly symmetric. In addition, by increasing the number of barriers, the resonant peaks become close to each other and form minibands of resonances separated by gaps. The size of these minibands increases with the thickness of the barriers. 

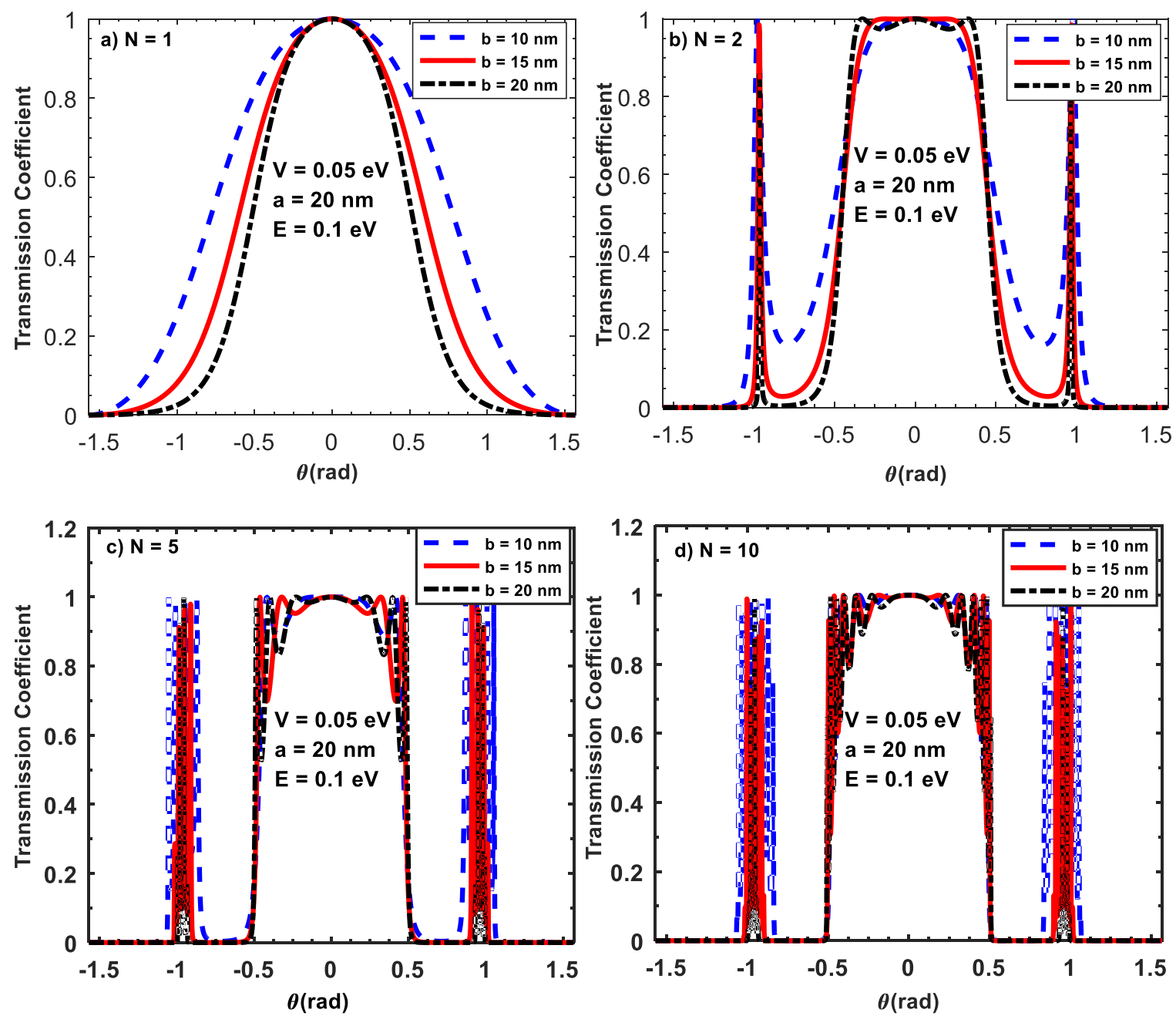

Figure 4. Variation of the transmission coefficients as a function of the angle of incidence for different barrier widths. a) $N=1$, b) $N=2$, c) $N=5$, and d) $N=10$

The electronic conductance $G\left(G_{0}\right)$ as a function of the Fermi level is displayed in figures 5 (a-d). In these figures, we evaluate the conductance for $N=1,2,4$, and 5 . In this case, we consider a symmetric structure $(a=b=20 \mathrm{~nm})$ with the barrier height fixed to $V=0.05 \mathrm{eV}$. The conductance strongly changes when the number of barriers at low Fermi energies is increased. For instance, when we consider one barrier $(N=1)$, the conductance has only one peak in the Fermi energy region below the height barrier $\left(E_{F}<0.05 \mathrm{eV}\right)$. However, additional peaks appear for $N=$ 2 , 4, and 5 as shown in figures 5 (b-d). For Fermi energies above the barrier height $E_{F}>0.05 \mathrm{eV}$, the conductance is strongly decreased as the Fermi energy increases, and the resonant feature disappears. In addition, the conductance presents the same variation regardless of the number of barriers in the higher Fermi level regions $\left(E_{F}>0.1 \mathrm{eV}\right)$. 


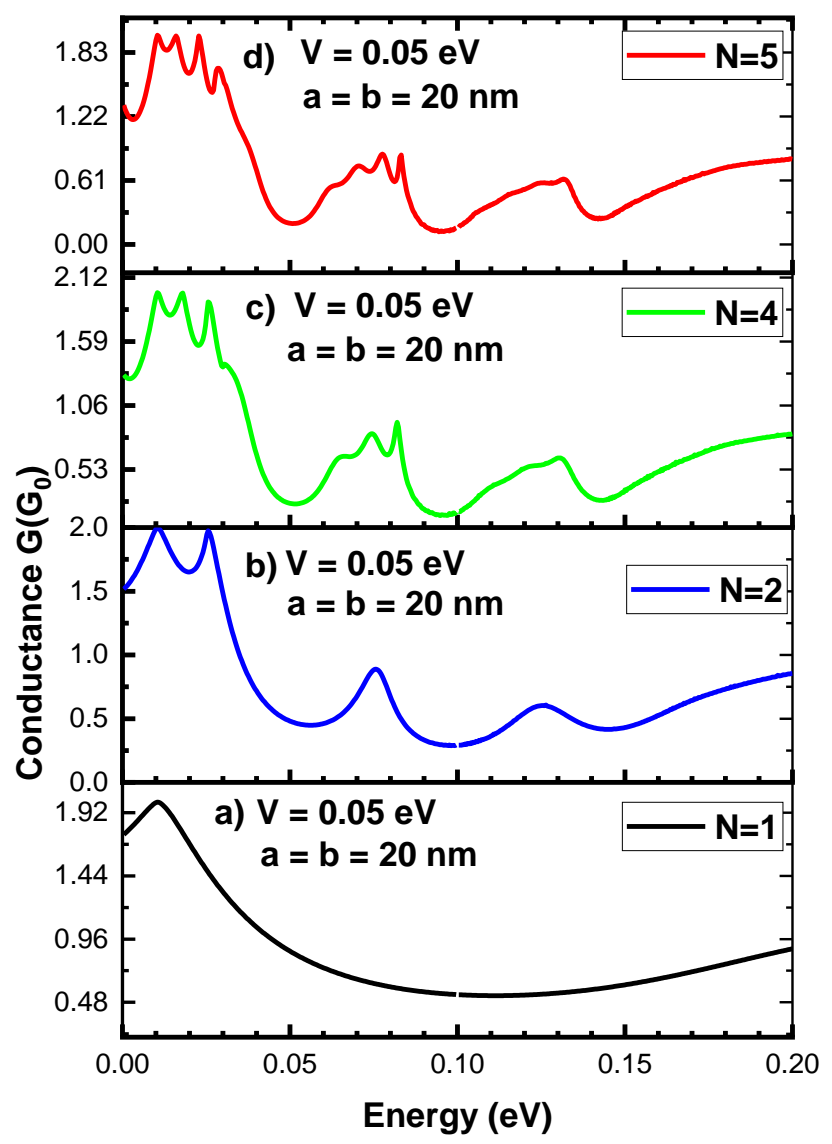

Figure 5. Variation of the electronic conductance as a function of the Fermi energy for different number of barriers. a) $N=1$, b) $N=2$, c) $N=4$, and d) $N=5$.

The dependence of the conductance on the widths of barriers is given in figure $6(\mathrm{a}-\mathrm{c})$, where three barrier widths are examined $(b=10,15$, and $20 \mathrm{~nm})$. Here, we considered 5 barriers separated by an inter-barrier distance of $a=20 \mathrm{~nm}$. For Fermi energies lower than the barrier height $\left(E_{F}<V=0.05 \mathrm{eV}\right)$, the transmission of electrons is governed by a Klein tunneling phenomenon which predicts that the electrons are resonantly transmitted across the barrier via confined hole states. This phenomenon produces resonant peaks in the conductance whose number increases with the barrier width. Furthermore, we note that by increasing the barriers widths, the conductance minima move towards the right for lower Fermi energies $\left(E_{F}<0.05 \mathrm{eV}\right)$ and towards the left for higher Fermi energies $\left(E_{F}>0.05 \mathrm{eV}\right)$. 


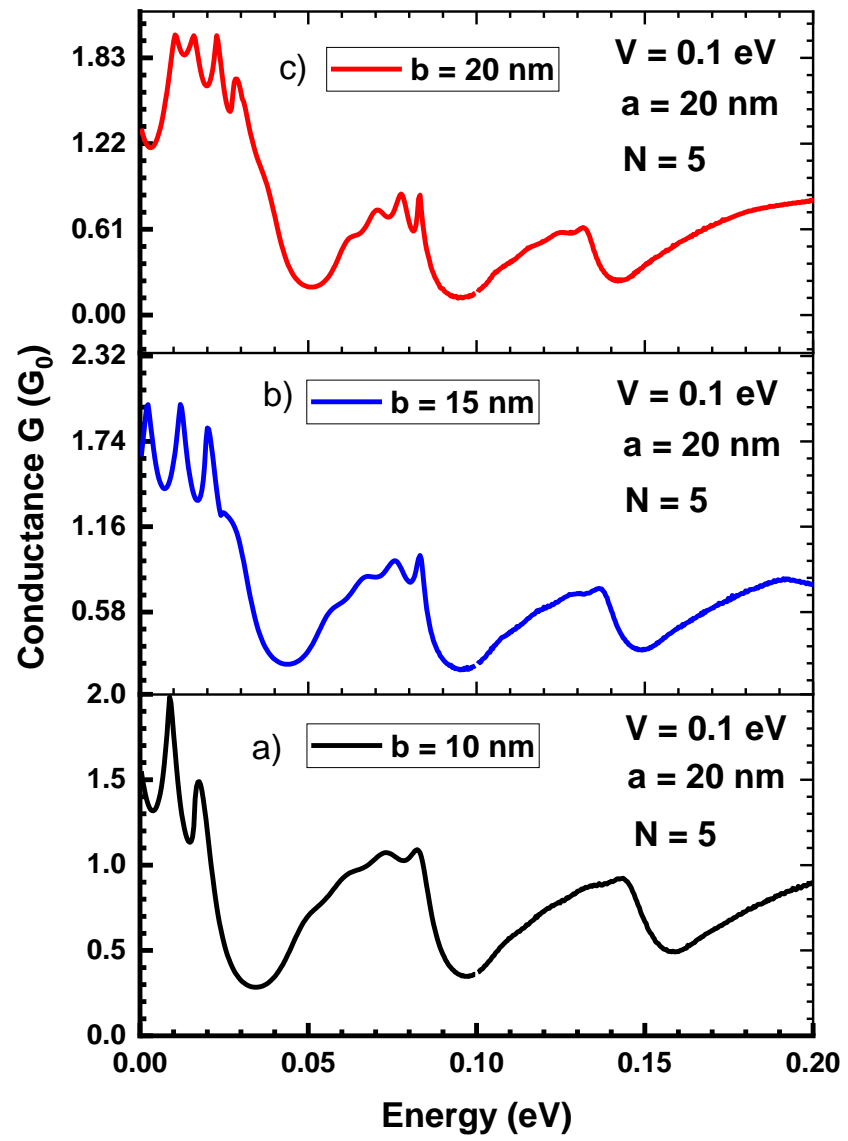

Figure 6. Variation of the electronic conductance as a function of the Fermi energy for different widths of barriers. a) $N=1$, b) $N=2$, c) $N=4$, and d) $N=5$.

To conclude our analysis, figure 7 (a-c) calculates the Fano factor as a function of the Fermi energy. The physical parameters are the same as those used in figure 6 . The Fano factor shows oscillatory regions separated by inverse-parabolic shapes that correspond to the minima observed in the conductance (figure 6). In other words, the Fano resonance factor reaches its maximum when the conductance is at its minimum and vice versa. This behavior arises since the Fano factor is the inverse of the conductance. Furthermore, the energy separations between the peaks of the Fano factor match the widths of the energy gap regions observed in the variation of the conductance. In addition, we observe that the maxima of the Fano factor are not affected by increasing the widths of the barriers. The only effect of enlarging barrier width is that the energy gaps in the Fano factor become wider. 


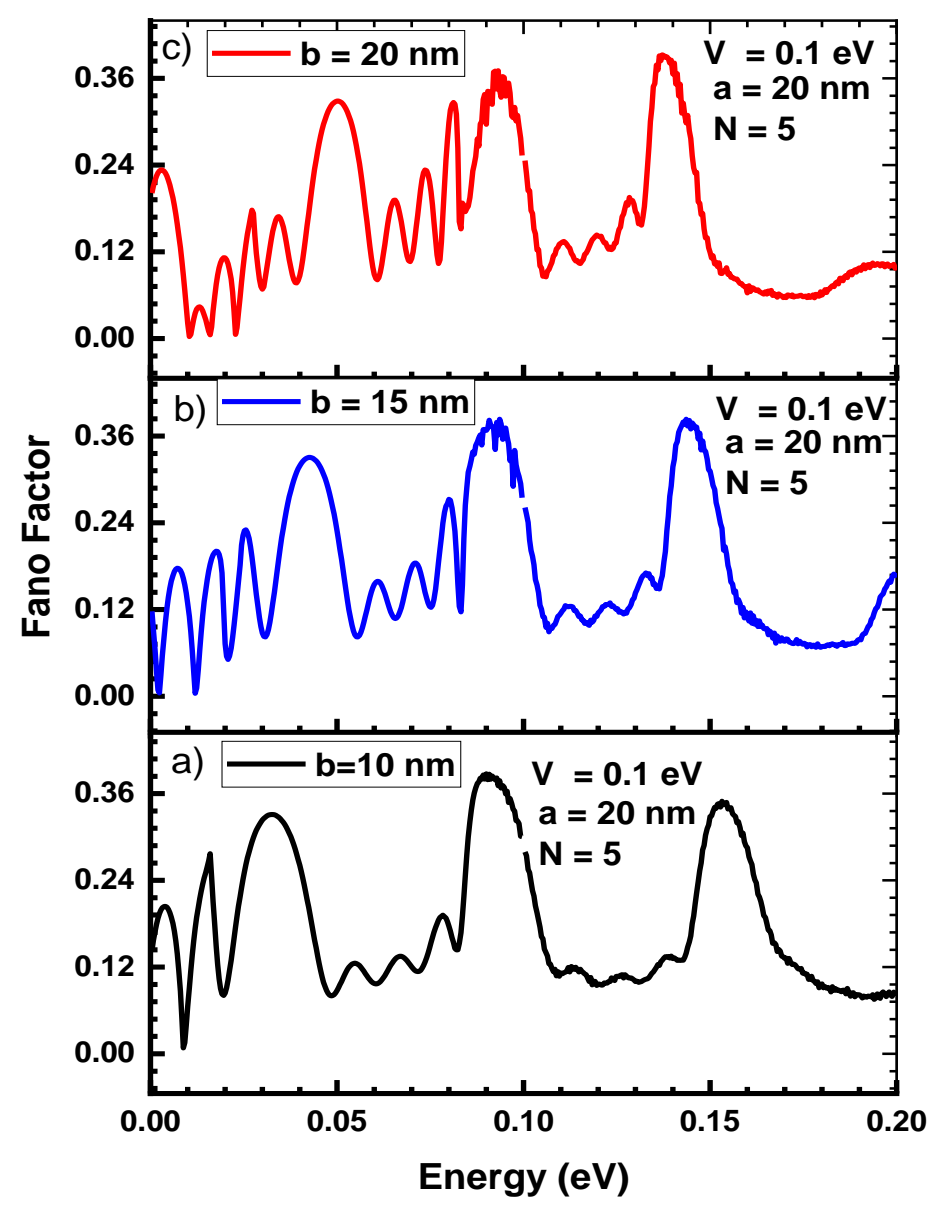

Figure 7. Variation of the Fano Factor as a function of the Fermi energy for different widths of barriers. a) $b=10 \mathrm{~nm}$, b) $b=15 \mathrm{~nm}$, and c) $b=20 \mathrm{~nm}$

\section{Conclusion}

In summary, we have studied the electronic transmission and conductance of Dirac electrons across an electrostatic multibarrier deposited on monolayer graphene. The transfer matrix method (TMM) was employed to compute the transmission coefficient for different numbers and widths of barriers as well as various angles of incidence. From these results, we calculated the electronic conductance and Fano factors. We have shown that the number of resonant states is proportional to the number of barriers. For a large number of barriers $(N=10)$, the transmission coefficient presents welldefined miniband regions separated by energy gaps. In addition, by increasing the barrier width, the conductance shifts to the right at lower incident energies and shifts to the left at higher ones. These interesting findings assist in interpreting experimental phenomena in graphene-based structures as well as constructing various optoelectronic devices such as the field-effect transistors based on (ON/OFF) states. 


\section{Acknowledgements}

B.M.W. acknowledges financial support from the Office of Naval Research (Grant N00014-18-12740).

\section{References}

[1] K. S. Novoselov, A. K. Geim, S. V. Morozov, D. Jiang, Y. Zhang, S. V. Dubonos, I.V.Grigorieva, A. A. Firsov, Science 306 (2004) 666-669.

[2] A. H. CastroNeto, F. Guinea, N. M. R. Peres, K. S. Novoselov, A. K. Geim, Reviews of Modern Physics 81 (2009) 109-162.

[3] C. Berger, Z. M. Song, X. B. Li, X. S. Wu, N. Brown, C. Naud, D. Nato, T. B. Li, J. Hass, A. N. Marchenkov, E. H. Conrad, P. N. First, W. A. de Heer, Science312(2006)1191-1196.

[4] Y. Zhang,Y. W. Tan, H. L. Stormer, P. Kim, Nature 438 (2005) 201-204.

[5] M. I. Katsnelson, K. S. Novoselov, A. K. Geim, Nature Physics 2 (2006) 620-625.

[6] K.S. Novoselov, A.K. Geim, S.V. Morozov, D. Jiang, M.I. Katsnelson, I.V. Grigorieva, S.V. Dubonos, A.A. Firsov, Nature 438 (2005) 197-200.

[7] J. Tworzydlo, B. Trauzettel, M. Titov, A. Rycerz, C.W.J. Beenakker, Physical Review Letters 96 (2006) 246802-246805.

[8] B.L. Zhou, B.H. Zhou, W.H. Liao, G.H. Zhou, Physics Letters A 374 (2010) 761-764.

[9] R. Zhu, Y. Guo, Applied Physics Letters 91 (2007) 252113-252115.

[10] A. De Martino, L. Dell'Anna, R. Egger, Physical Review Letters 98 (2007) 066802-066805.

[11] M. Tahir, K. Sabeeh, Physical Review B 77 (2008) 195421-195425.

[12] J.J. MiltonPereira, P. Vasilopoulos, F.M. Peeters, Applied Physics Letters 90 (2007) 132122132124.

[13] M.R. Masir, P. Vasilopoulos, A. Matulis, F.M. Peeters, Physical Review B 77 (2008) 235443235453.

[14] L. Dell'Anna, A. De Martino, Physical Review B 79 (2009) 045420-045428.

[15] X. L. Lu, Y.S.Zheng,H.W.Xin,L.W.Jiang,Applied Physics Letters 96 (2010) 132108132110.

[16] G. J. Xu, X. G. Xu, B. H. Wu, J. C. Cao, C. Zhang, Journal of Applied

Physics 107(2010)123718-123724.

[17] T. X. Ma,C. Liang, L. G. Wang, H. Q. Lin, Applied. Physics. Letters. 100

(2012) 252402-252405. 
[18] H. Y. Zhang, Y. Gao, Y. P. Zhang, S. L. Xu, S. F. Wang, Applied Physics

Letters 99(2011)072108-072110.

[19] F. M. D. Pellegrino,G. G. N. Angilella, R. Pucci, Physical Review B 84

(2011) 195404-195414.

[20] N. Abedpour, A. Esmailpour, R. Asgari, M. R. R. Tabar, Physical

Review B79(2009)165412-165418.

[21] R. Biswas,A. Biswas,N. Hui,C. Sinha, Journal of Applied Physics 108

(2010) 043708-043714.

[22] M. Ramezani Masir, P. Vasilopoulos, F. M. Peeters, Applied Physics. Letters. 93 (2008)

242103-242105.

[23] Y. P. Bliokh, V. Freilikher, F. Nori, Physical Review B. 81 (2010) 075410-075418.

[24] G. W. Semenoff, Physical Review Letters 53 (1984) 2449-2452.

[25] F. Zhai, K. Chang, Physical Review B 77 (2008) 113409-113412.

[26] J. Tworzydlo, B. Trauzettel, M. Titov, A. Rycerz, C.W.J. Beenakker, Physical Review Letters 96 (2006) 246802-246805.

[27] M. I. Katsnelson, European Physical Journal B 51 (2006) 157-160.

[28] M. Titov, Europhysics Letters 79 (2007) 17004-17009.

[29] M. Buttiker, Y. Imry,R. Landauer,S. Pinhas, Physical Review B 31 (1985) 6207-6215;

[30] J. R. F. Lima, L. F. C. Pereira, and C. G. Bezerra. J. Appl. Phys. 119 (2016) 244301.

[31] Y. Joo, M. Kim, P. Huang, C. Kanimozhi, B. M. Wong, S. S. Roy, M. S. Arnold, and P. Gopalan, Journal of Physical Chemistry C (2016) 120, 13815-13824

[32] P. S. Johnson, C. Huang, M. Kim, N. S. Safron, M. S. Arnold, B. M. Wong, P. Gopalan, and F. J. Himpsel, Langmuir (2014) 30, 2559-2565

[33] C. Huang, M. Kim, B. M. Wong, N. S. Safron, M. S. Arnold, and P. Gopalan, Journal of Physical Chemistry C (2014) 118, 2077-2084 